\title{
Delayed complications and functional outcome of isolated sternal fracture after emergency department discharge: a prospective, multicentre cohort study
}

\author{
Samuel Racine, $\mathrm{MD}^{* * *}$; Marcel Émond, $\mathrm{MD}, \mathrm{MSc}^{*}{ }^{* *}$; Jean-Sébastien Audette-Côté, MD"; \\ Natalie Le Sage, MD, $\mathrm{PhD}^{* * * *}$; Chantal Guimont, MD, $\mathrm{PhD}^{+* *}$; Lynne Moore, $\mathrm{PhD}^{* * *}$; Jean-Marc \\ Chauny, MD, MSc ${ }^{\ddagger}$; Éric Bergeron, MD, MSc ${ }^{* \S}$; Laurent Vanier, MD, $\mathrm{PhD}^{\S}$
}

\section{ABSTRACT}

Objective: The aim of this study was to determine the incidence of delayed complications, specifically hemothorax, and functional outcome in patients with isolated sternal fracture discharged from the emergency department (ED) compared to patients with other minor thoracic trauma.

Methods: This prospective cohort study was conducted in four university-affiliated Canadian EDs. Patients ages 16 and older discharged from the ED with an isolated minor thoracic injury were included and categorized as isolated sternal fracture, rib fracture, or no fracture. A standardized clinical and radiological follow-up was performed at 7 and 14 days as well as a phone follow-up at 30 and 90 days post-injury. Functional outcome was determined using the Medical Outcome Short-Form Health Survey (SF-12).

Results: A total of 969 patients were included, of whom 32 (3.3\%) had an isolated sternal fracture, 304 (31.3\%) had rib fracture, and $633(65.3 \%)$ had no fracture. Within 14 days, 112 patients presented with a delayed hemothorax: $12.5 \%$ of sternal fracture patients, $23 \%$ of rib fracture(s) patients, and $6 \%$ of minor thoracic injury patients without fracture $(p<0.05)$. At 90 days, $57.1 \%$ of patients with sternal fracture had moderate to severe disability compared to $25.4 \%$ and $21.2 \%$ for both of the other groups, respectively $(p<0.001)$.

Conclusion: In this prospective study, we found that $12.5 \%$ ( $n=4, p<0.05$ ) of patients with sternal fracture developed a delayed hemothorax, but the clinical significance of this remains questionable. The proportion of patients with sternal fracture who had moderate to severe disability was significantly higher than that of patients with other minor thoracic trauma.

\section{RÉSUMÉ}

Objectif: L'étude visait à déterminer l'incidence de complications tardives, précisément de I'hémothorax, et de résultats cliniques fonctionnels chez des patients ayant subi une fracture isolée du sternum, et ce après le congé du département d'urgence (DU), comparativement à des patients ayant subi d'autres traumas thoraciques mineurs.

Méthode: II s'agit d'une étude prospective de cohorte qui a été menée dans quatre DU rattachés à des centres hospitaliers universitaires au Canada. Des patients âgés de 16 ans et plus ayant obtenu leur congé du DU après avoir subi un traumatisme thoracique mineur isolé ont participé à cette étude; leur état a été catégorisé comme suit : fracture isolée du sternum, fracture(s) de côte(s) ou aucune fracture. Un suivi clinique et radiologique standardisé a été effectué au $7^{\mathrm{e}}$ et $14^{\mathrm{e}}$ jour, ainsi qu'un suivi téléphonique au $30^{\mathrm{e}}$ et $90^{\mathrm{e}}$ jour après l'accident. Les résultats cliniques fonctionnels ont été évalués selon le questionnaire Medical Outcomes Short-Form Health Survey (SF-12).

Résultats: Au total, 969 patients ont participé à l'étude, dont $32(3,3 \%)$ souffraient d'une fracture isolée du sternum; 304 $(31,3 \%)$, d'une fracture(s) de côte(s) et $633(65,3 \%)$, d'aucune fracture. Au cours des 14 jours suivants, 112 patients ont consulté pour un hémothorax retardé : $12,5 \%$ à la suite d'une fracture du sternum; $23 \%$ à la suite d'une fracture(s) de côte(s) et $6 \%$ à la suite de traumatisme thoracique mineur sans fracture $(p<0,05)$. Au bout de 90 jours, $57,1 \%$ des patients ayant subi une fracture du sternum présentaient une incapacité modérée ou grave par rapport à $25,4 \%$ et $21,2 \%$ dans les deux autres groupes, respectivement $(p<0,001)$.

Conclusions: Les résultats de cette étude prospective démontrent qu'un hémothorax retardé s'est formé chez $12,5 \%(\mathrm{n}=4 ; p<0,05)$ des patients ayant subi une fracture du sternum, mais la portée clinique de cette complication reste discutable. Une incapacité modérée ou grave a été observée dans une proportion significativement plus grande de patients ayant subi une fracture du sternum que de patients ayant subi d'autres traumas thoraciques mineurs.

From the *Axe santé des populations et pratiques optimales en santé, Centre de recherche du CHU de Québec, Hôpital de l'Enfant-Jésus, Québec, QC; †Département de médecine familiale et de médecine d'urgence, Université Laval, Québec, QC; ¥Centre hospitalier du Sacré-Cœur de Montréal, Montréal, QC; §Centre hospitalier de Charles-LeMoyne, Greenfield Park, Longueuil, QC; ๆCentre hospitalier régional de Lanaudière, Joliette, QC; and; and ${ }^{*} \mathrm{CHU}$ de Québec, Québec, QC.

Correspondence to: Dr. Marcel Émond, Emergency Department, CHU de Québec, Hôpital de l'Enfant-Jésus, 1401, 18e Rue, Room H-607, Québec, OC G1J 1Z4; Email: marcelemond1@me.com. 
Keywords: isolated sternal fracture, minor thoracic trauma, delayed complications, funtionnal outcome, delayed hemothorax

\section{INTRODUCTION}

Sternal fracture is usually the result of significant blunt thoracic trauma and occurs more frequently in drivers wearing three-point restraint who are involved in a motor vehicle crash. ${ }^{1}$ Its reported incidence varies between $0.33 \%$ of trauma patients ${ }^{2}$ and $3.7 \%$ of patients admitted after a motor vehicle $\mathrm{crash}^{3}$ and increases with age. It can be present in up to $27.7 \%$ of thoracic injuries associated with motor vehicle crashes in the extreme elderly population ( $>80$ years of age). ${ }^{4}$ Sternal fractures are often associated with other injuries, most commonly soft tissue contusions $(56 \%)$ and rib fractures $(49.6 \%){ }^{2}$ When isolated, they are associated with a reported mortality rate of between $0.7 \%$ and $3.5 \%$. Poor outcomes are associated with the severity of other injuries, complications, and pre-existing comorbidity. ${ }^{3,5}$

Knowledge regarding the clinical significance of isolated sternal fracture has evolved, and many studies have demonstrated that patients with this injury can safely be discharged from the emergency department (ED) after investigation. ${ }^{6-9}$ A retrospective cohort study found that cardiac complications were present in $0.8 \%$ of isolated sternal fracture cases. ${ }^{5}$ Clinically significant cardiac complications associated with isolated sternal fracture have been shown to be essentially nonexistent when an initial electrocardiogram and cardiac biomarker levels are normal. ${ }^{7}$ Current literature suggests that routine admission is no longer required for isolated sternal fracture ${ }^{9,10}$; however, this practice remains widespread. ${ }^{11}$

Delayed hemothorax has been reported as an important complication with patients with minor thoracic injuries discharged from the ED, with an incidence of $7.4 \%$ of blunt thoracic injuries reported in Europe ${ }^{12}$ and $11.8 \%$ in North America. ${ }^{13}$ However, the only published prospective studies addressing delayed complications of minor thoracic injuries did not specifically assess isolated sternal fractures. ${ }^{12-14}$ Moreover, very few authors have studied functional outcome or pain experienced by patients with minor thoracic injuries or sternal fracture after ED discharge. ${ }^{14-17}$

The purpose of this study was to determine and compare complications and outcomes of patients with isolated sternal fracture discharged from the ED to those with other minor thoracic injury.

\section{METHODS}

\section{Study setting and population}

This prospective observational cohort study was conducted in four Canadian university-affiliated EDs (Hôpitaux l'Enfant-Jésus and CHUL of the CHU de Québec, Centre Hospitalier du Sacré-Cœur de Montréal, Centre hospitalier Charles-Lemoyne), between March 25, 2006 and November 2, 2010 and was a preplanned subanalysis of a larger project with prior publications involving the follow-up of patients with minor thoracic injury. ${ }^{13,14,17}$ Patients ages 16 years and over who presented to the ED with a minor thoracic injury were screened for eligibility. Minor thoracic injury was defined by the presence of chest abrasion or contusion, or suspected or radiographically confirmed rib fracture. All participants were discharged from the ED after receiving care by the attending physician (primary care physician, emergency physician, or other specialist). Patients were excluded if 1) they had a hemothorax, pneumothorax, lung contusion, or any significant cerebral, thoracic, abdominal, or extremity injury diagnosed on their initial ED visit; or 2) a followup was not possible due to geographic or social circumstances; or 3) there was a delay greater than 3 days between the injury and the ED visit (a cut-off based on a previous publication showed that $90 \%$ of patients seeking medical attention in an acute care setting do so within 72 hours of injury). ${ }^{18}$ The study was approved by the Research Ethics Board of all four involved institutions, and written consent was obtained from study participants.

\section{Study protocol}

Initial ED care, evaluation, and data collection were left to the discretion of the attending physicians, who were blinded to the research protocol and study outcomes. Participants thus received usual care and standard discharge instructions. Participants then returned for standardized medical outpatient evaluations at 7 and 14 days post-injury. Assessment at these visits included pain severity, clinical examination, and an upright chest $\mathrm{X}$-ray. There was no specific protocol regarding pain regimen, and this, too, was left to the discretion of the physician involved. Information on potentially significant delayed complications, including hemothorax, pneumothorax, atelectasis, and lung contusion, were 
obtained from the radiology report. Research nurses performed subject telephone interviews at 30 and 90 days post-injury, during which they captured data on severity of pain, functional status, and complications.

\section{Primary outcomes}

Delayed hemothorax was deemed present if pleural fluid was identified on the follow-up chest X-ray. A blinded radiologist made the final interpretation of these radiographs, and the size of hemothorax was not assessed. The decision on whether to treat a hemothorax was left to the discretion of the attending physician.

Functional status was assessed at 30 and 90 days, using the Medical Outcome Short-Form Health Survey (SF-12), a validated instrument derived from the more extensive SF-36 health survey. ${ }^{19}$ It contains 12 questions that address 8 health elements: physical functioning in daily activities, role limitations due to physical health problems, overall bodily pain limiting usual activities, physical and mental health summary score, energy/fatigue, social functioning, and role limitation due to emotional health problems. Scores for each element range on a scale of $0-100$, where 0 is the worst outcome and 100 is the best. Two aggregated summary scores (0-100) can also be derived: a global physical health summary (PHS) score and a global mental health summary (MHS) score. PHS, MHS, role limitations, physical functioning, and overall bodily pain were retained for analysis in our cohort and are presented in the results section. In order to make the interpretation of the outcome easier, disability was defined according to PHS and MHS scores that were normalized for age and gender of a North American population and categorized into none/mild (>40), moderate (25-40), and severe disability $(<25)$, according to previous publications. ${ }^{20,21}$

\section{Data analysis}

Clinical and demographic characteristics were described using univariate methods. We used the chi-square test for categorical variables and student t-test for continuous variables to assess primary and secondary outcomes. Comparisons of mean differences of the PHS scores adjusted for age, gender, and mechanism of injury, and presence of pulmonary comorbidities between categories at 90 days was performed using analysis of variance, also known as ANOVA. Correlation adjustment and Scheffé's multiple test comparisons methods were included. Data analyses were performed using SAS 9.2 for Windows (SAS Institute, Gary, NC, USA).

\section{RESULTS}

A total of 2866 patients were screened for eligibility during the study period, and 969 were retained for analysis, as shown in Figure 1. Table 1 provides demographic and clinical characteristics of the study groups and illustrates the mean age of our study population$52.9( \pm 16.7)$ years old ranging from 16 to 95 . Participants were slightly younger in the group of patients with minor thoracic injuries without fracture than in other groups, $50.9( \pm 17.5)$ versus $55.2( \pm 16.2)$ for sternal fracture group and $56.3( \pm 14.4)$ for rib fracture(s) group $(p<0.001)$. Fifteen patients $(46.9 \%)$ with isolated sternal fracture were age 65 or more. There were more males in the rib fracture(s) and thoracic injury without fracture groups than in the sternal fracture group $(69.4 \%$ v. $60.2 \%$ v. $46.9 \%)$. Motor vehicle crash was the principal mechanism of injury (78\%) in the sternal fracture group in contrast to falls in both of the other groups. One patient presenting with a sternal fracture associated with at least one rib fracture and was excluded from the analysis. This patient did not have delayed hemothorax identified at follow-up. Thirty-two patients (3\%) had an isolated sternal fracture.

Table 2 provides information on delayed complication in the study population. Overall, 112 patients presented with a hemothorax within 14 days postinjury. The incidence of delayed hemothorax was $12.5 \%$ in the sternal fracture group $(n=4), 23 \%$ in the rib fracture(s) group $(\mathrm{n}=70)$, and $6 \%$ in the minor thoracic injury without fracture(s) group $(\mathrm{n}=38)$, $(p<0.05)$. All delayed hemothoraces in the sternal fracture group were detected before day 7 , as were the majority of delayed hemothoraces in both of the other groups. One delayed hemothorax in the rib fracture group was detected after 14 days but kept for analysis in that group. None of the delayed hemothoraces in the isolated sternal fracture and thoracic injury without fracture groups required drainage. Three of the delayed hemothoraces in the rib fracture group were managed with drainage. The four participants in the sternal fracture group who developed delayed hemothorax were aged 53, 71, 73, and 75 years. One of those patients was taking aspirin, and none were on other antiplatelet or anticoagulant medications. Atelectasis 


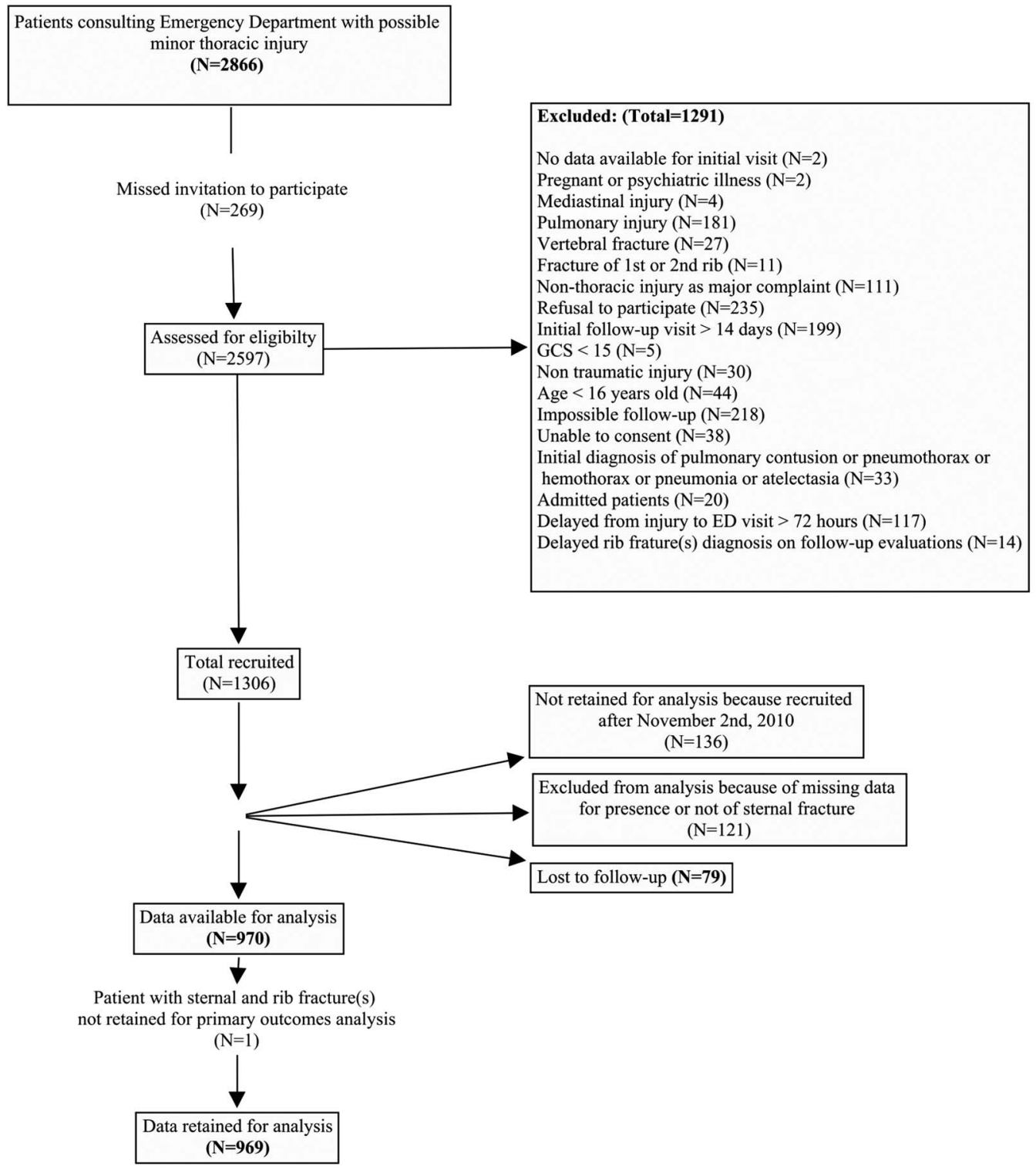

Figure 1. Flow diagram.

was the only other delayed complication observed on follow-up radiographs in the sternal fracture group. No cases of pneumonia or pneumothorax were detected in the sternal fracture group, and few were present in both of the other groups. There were no cardiac complications listed, because patients were assessed and excluded at initial evaluation if such complications were present. No mortality was found in any participants at the 90-day follow-up.

Isolated sternal fracture patients had more important physical disability on SF-12 testing at 30 days (mean, SD: $30.6 \pm 11.2)$ compared to $35.2 \pm 10.5$ and $39.6 \pm$ 12.6 for both of the other groups, respectively $(p<0.001)$, as shown in Figure 2, A. At 90 days, only $42.9 \%$ of patients with sternal fracture had little to no disability, in comparison to $74.6 \%$ and $78.8 \%$ of patients from other groups $(p<0.01)$, as shown in Figure 2, B. Of sternal fracture patients, $57.1 \%$ had moderate to severe disability compared to $25.4 \%$ and $21.2 \%$, respectively, for both of the other groups $(p<0.001)$. When taken separately, severe functional impairment was not statistically significant between groups. No significant difference was found between groups regarding the psychological disability. The incidence of a severe mental disability was low in all three groups at 90 days $(0.0 \%$ for sternal fracture v. $3.2 \%$ 


\begin{tabular}{|c|c|c|c|c|c|}
\hline Characteristic & $\begin{array}{c}\text { Total } \\
\left(\mathrm{N}^{\mathrm{a}}=969^{\mathrm{b}}\right) \%\end{array}$ & $\begin{array}{l}\text { Sternal fracture } \\
\left(\mathrm{N}^{\mathrm{a}}=32\right) \%\end{array}$ & $\begin{array}{l}\text { Rib fracture(s) } \\
\left(\mathrm{N}^{\mathrm{a}}=304\right) \%\end{array}$ & $\begin{array}{l}\text { Thoracic injury without fracture } \\
\qquad\left(N^{a}=633\right) \%\end{array}$ & $P$-value \\
\hline Age (mean, SD) & $(52.9,16.7)$ & $(55.2,16.2)$ & $(56.3,14.4)$ & $(50.9,17.5)$ & $<0.001$ \\
\hline Age $>65$ years old & 26.2 & 40.6 & 28.0 & 24.6 & 0.09 \\
\hline Gender (male) & 62.6 & 46.9 & 69.4 & 60.2 & 0.003 \\
\hline \multicolumn{6}{|l|}{ Mechanism of injury } \\
\hline Fall of own height & 33.5 & 6.3 & 38.5 & 32.5 & \multirow[t]{7}{*}{$<0.001$} \\
\hline Fall exceeding own height & 21.5 & 3.1 & 29.3 & 18.7 & \\
\hline Motor vehicle crash & 27.1 & 78.1 & 16.1 & 29.8 & \\
\hline Pedestrian v. auto & 1.2 & 0.0 & 2.0 & 1.0 & \\
\hline Bicyclist v. auto & 0.9 & 0.0 & 1.3 & 0.8 & \\
\hline Direct impact & 10.3 & 12.5 & 8.6 & 11.1 & \\
\hline Other & 5.5 & 0.0 & 4.3 & 6.3 & \\
\hline \multicolumn{6}{|l|}{ Pre-existing co-morbidity } \\
\hline $\begin{array}{l}\text { Presence of at least } 1 \text { pre-existing } \\
\text { co-morbidity }\end{array}$ & 25.1 & 21.9 & 22.7 & 26.4 & 0.43 \\
\hline $\begin{array}{l}\text { Airway disease, asthma and/or } \\
\text { COPD }\end{array}$ & 10.5 & 12.5 & 6.6 & 12.3 & 0.03 \\
\hline Osteoporosis & 7.3 & 3.1 & 7.2 & 7.6 & 0.64 \\
\hline \multicolumn{6}{|l|}{ Smoking status } \\
\hline Current smoker & 30.6 & 14.3 & 31.9 & 30.9 & \multirow[t]{3}{*}{0.21} \\
\hline Former smoker & 25.1 & 28.6 & 27.6 & 23.7 & \\
\hline Non-smoker & 44.3 & 57.1 & 40.5 & 45.4 & \\
\hline \multicolumn{6}{|l|}{ Anticoagulant medication } \\
\hline ASA & 16.5 & 12.5 & 16.8 & 16.6 & 0.8 \\
\hline Clopidogrel & 1.6 & 0.0 & 1.3 & 1.7 & $0.9^{*}$ \\
\hline Warfarin & 4.0 & $2(6.3)$ & $7(2.3)$ & $30(4.7)$ & 0.17 \\
\hline \multicolumn{6}{|l|}{ Physical evaluation } \\
\hline Pleuritic chest pain & 53.6 & 34.4 & 55.9 & 53.4 & 0.07 \\
\hline Severe chest pain & 53.8 & 46.2 & 61.3 & 50.7 & 0.02 \\
\hline Hemoptysis & 0.8 & 3.1 & 0.7 & 0.8 & $0.3^{*}$ \\
\hline Dyspnea at rest & 6.8 & 6.3 & 8.9 & 5.9 & 0.2 \\
\hline Chest abrasion & 5.1 & 3.1 & 3.3 & 6.0 & 0.2 \\
\hline Chest contusion & 19.2 & 12.5 & 14.5 & 21.8 & 0.02 \\
\hline \multicolumn{6}{|l|}{ Vital signs (mean, SD) } \\
\hline Pulse $>100$ & 9.1 & 6.3 & 8.0 & 9.8 & 0.6 \\
\hline Oxygen saturation $\leq 92 \%$ & 0.8 & 0.0 & 0.3 & 1.0 & $0.6^{*}$ \\
\hline Respiratory rate $\geq 20 / \mathrm{min}$ & 33.8 & 41.9 & 38.2 & 31.2 & 0.08 \\
\hline \multicolumn{6}{|l|}{ Number of confirmed rib fractures } \\
\hline At least 1 & 31.4 & - & 100.0 & - & \\
\hline At least 2 & 17.1 & - & 54.6 & - & \\
\hline 3 and more & 8.2 & - & 26.0 & - & \\
\hline \multicolumn{6}{|c|}{$\begin{array}{l}\text { ASA = Aspirin; } \mathrm{COPD}=\text { chronic obstructive pulmonary disease; } \mathrm{SD}=\text { standard deviation. } \\
\text { Chi-square test for categorical variables, and test of student for continuous variables. } \\
\text { aBecause of missing data, the number of patients does not always add to the total. } \\
\text { bOne patient presented with a sternal fracture and at least one rib fracture and was excluded from primary outcomes analysis and thus not included. } \\
\text { "Fisher exact test. } \\
\text { Mean values of pulse, oxygen saturation, blood pressure, and respiratory rate were similar across groups. Missing data exist for mechanism of injury }(n=1) \text {, smoking status }(n=169) \text {, severe } \\
\text { chest pain }(n=200) \text {. }\end{array}$} \\
\hline
\end{tabular}

for rib fracture(s) v. $4.2 \%$ for minor thoracic injury without fracture $(p=0.8))$. A comparison of mean PHS score differences at 90 days is presented in Table 3 and shows that sternal fracture was associated with overall significantly lower global mean PHS scores. The sternal fracture group had a lower score when compared with both the rib fracture(s) and the no fracture group. 


\begin{tabular}{|c|c|c|c|c|c|}
\hline Complication & $\begin{array}{l}\text { Total } \% \\
(\mathrm{~N}=969)\end{array}$ & $\begin{array}{l}\text { Sternal fracture \% } \\
\qquad(\mathrm{N}=32)\end{array}$ & $\begin{array}{l}\text { Rib fracture(s) \% } \\
\qquad\left(\mathrm{N}^{\mathrm{a}}=304\right)\end{array}$ & $\begin{array}{l}\text { Thoracic injury without } \\
\text { fracture } \%(N=633)\end{array}$ & $P$-value \\
\hline Hemothorax & 11.6 & 12.5 & 23.0 & 6.0 & $<0.0001$ \\
\hline \multicolumn{6}{|c|}{ Detection time of hemothorax } \\
\hline 0-7 days & 9.6 & 12.5 & 18.8 & 5.0 & $<0.0001$ \\
\hline 8-14 days & 2.0 & 0.0 & 3.9 & 0.9 & $0.009^{*}$ \\
\hline Atelectasis & 16.1 & 15.6 & 26.6 & 11.1 & $<0.0001$ \\
\hline Pneumonia & 0.3 & 0.0 & 0.7 & 0.2 & $0.3^{*}$ \\
\hline Pneumothorax & 0.7 & 0.0 & 1.6 & 0.3 & $0.09^{*}$ \\
\hline $\begin{array}{l}\text { Hemothorax managed } \\
\text { with drainage }\end{array}$ & 0.3 & 0.0 & 1.0 & 0.0 & n/a \\
\hline
\end{tabular}

Mean scores for physical functioning in daily activities at 30 days were significantly lower for the sternal fracture group $(38.1 \pm 12.4$ v. $44.5 \pm 11.8$ v. $46.5 \pm 11.6$, $p<0.01$ ), as shown in Figure 3, A. A similar nonstatistically significant trend was seen at 90 days. At 30 and 90 days, role limitations due to physical health problems mean scores were also significantly lower in patients with sternal fractures, as shown in Figure 3, B. Scores at 30 and 90 days for overall bodily pain limiting usual activities were significantly lower for this group of patients. At 30 days, mean bodily pain score was $31.5 \pm 11.5$ for sternal fracture in comparison to $36.7 \pm 10.9$ and $40.1 \pm 12.7(p<0.001)$ for both of the other groups. Following the same pattern, at 90 days, mean bodily pain score was $41.0 \pm 15.3$ for sternal fracture in contrast to $48.9 \pm 10.7$ and $50.9 \pm 10.3$ $(p<0.001)$ for both of the other groups.

Participants were deemed lost to follow-up if they had no visit within 14 days of initial assessment. Of the 969 initial participants, $79(8.1 \%)$ were lost to followup. Those participants were younger $(44.4 \pm 17.5)$, included a higher proportion of smokers, and presented with less severe dyspnea at rest at the initial evaluation compared to our participants. No other significant difference was found between patients lost to follow-up and the fully studied cohort. Data were available for 746 $(83.7 \%)$ and $624(70.0 \%)$ for the 30- and 90-day followup interviews, respectively. The number of participants followed at 30 days was approximately the same for each group $(90.9 \%$ for sternal fracture v. $94.1 \%$ for rib fracture(s) v. $95.7 \%$ for minor thoracic injury without fracture(s) $[p=0.4])$. Although the proportion of complete data at 90 days was lower in the sternal fracture group than in the other groups $(50 \%$ v. $79.7 \%$ and $79.9 \%, p<0.05)$, characteristics of the patients with missing data were similar in all three groups. No significant difference was found between participants with available data at 90 days and those with missing data in the sternal fracture group.

\section{DISCUSSION}

To our knowledge, this is the first prospective study to comparatively evaluate the complications and functional outcomes of isolated sternal fracture in trauma patients discharged from the ED. ${ }^{12-14}$ Current evidence suggests patients with isolated sternal fracture should not be admitted to the hospital, ${ }^{11}$ hence the relevance of our comparison of patients with isolated sternal fracture with those with minor thoracic injuries. The 3.2\% incidence of sternal fracture in our study population is similar to that reported for blunt thoracic injuries. ${ }^{2,3}$ We found that approximately one out of eight patients with an isolated sternal fracture developed a delayed hemothorax. This incidence was less than in the rib fracture(s) group, an injury known to be associated with delayed pulmonary complications, including hemothorax. ${ }^{13}$ None of the delayed hemothoraces in the sternal fracture group were managed with drainage, raising questions about the clinical significance of these radiologic findings. This situation could arise from the small number of isolated sternal fracture presenting with delayed hemothorax $(n=4)$ in our cohort, or could be that significant delayed hemothorax 
is rarely associated with isolated sternal fracture. Because the $11.6 \%$ incidence of all delayed hemothorax was slightly higher than previously reported, ${ }^{12,13}$ the
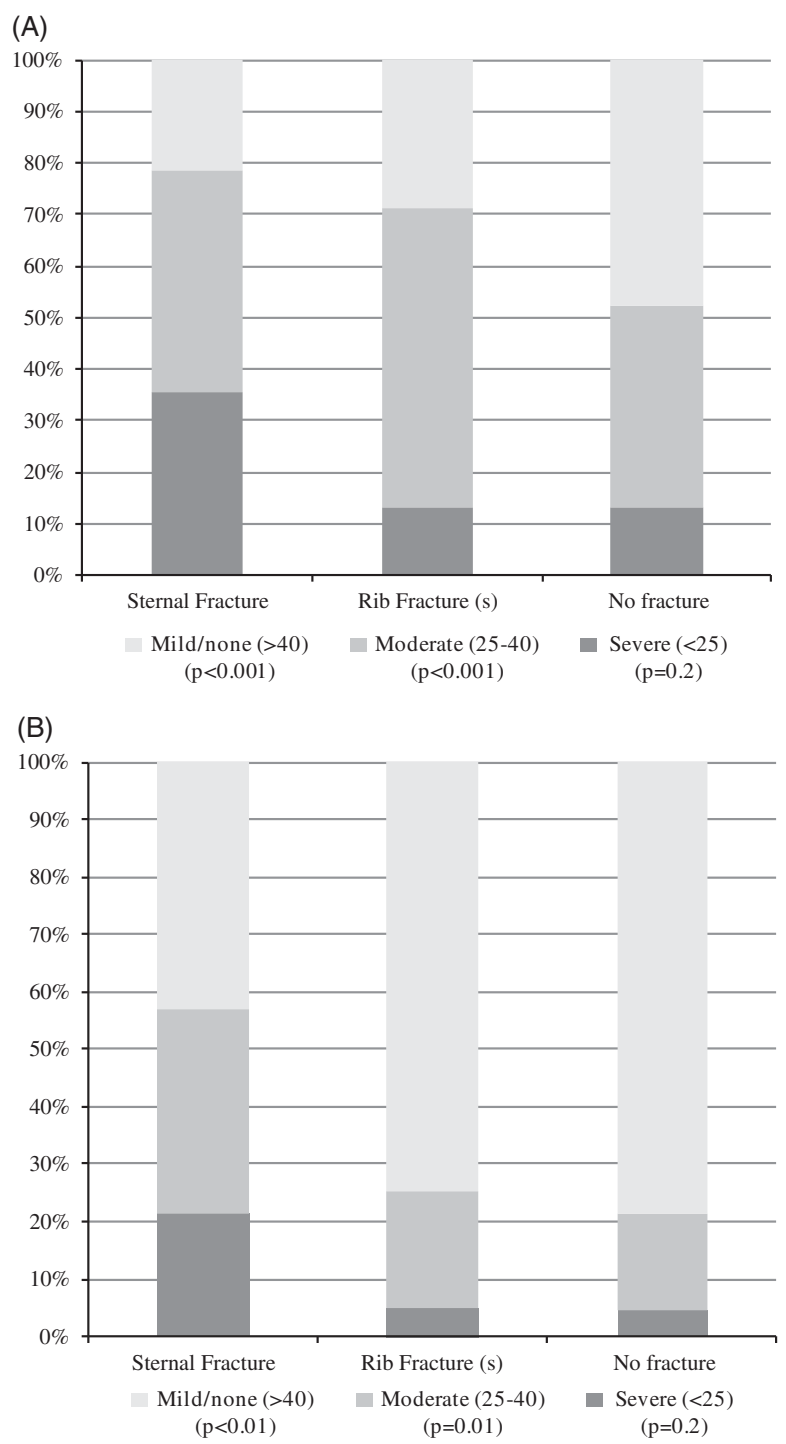

Figure 2. Functional outcomes according to SF-12 physical score. A) Functional outcomes at 30 days according to SF-12 physical score. B) Functional outcomes at 90 days according to SF-12 physical score. latter possibility seems more likely; however, further research would be required to answer this question definitively.

No previous study has reported on the functional impact of isolated sternal fracture on activities of daily living. It has been previously established that pain persists for a mean of 10.9 weeks and is significantly prolonged in patients over 50 years of age with isolated sternal fracture. ${ }^{16,17}$ Our results suggest that this pain can contribute to a major physical impairment. Indeed, when considering specific items of the SF-12 scale, such as physical functioning, role limitation, and overall bodily pain, patients with isolated sternal fracture were found to have more limitations than patients from other groups. It is possible that the functional limitations engendered by an isolated sternal fracture originated from the pain, delayed hemothorax, ${ }^{14}$ and global limitations in daily activities and roles. This possibility raises a concern of sufficient analgesia at discharge and emphasizes the importance of follow-up. It also corroborates some of our collaborators' conclusions that raised the importance of a better follow-up of patients with minor thoracic trauma, ${ }^{18}$ especially when delayed hemothorax is detected. ${ }^{14}$ Because sternal fractures do not seem to generate more psychological disability than other minor thoracic injuries, it seems logical that efforts should focus on the prevention of physical disability, which itself appears to lead to global functional impairment. Future studies should focus on factors that may increase the risk of developing a significant disability, analgesia at discharge, and the use of follow-up resources by patients with isolated sternal fracture.

The principal limitations of our study arise from missing data, in particular, in the sternal fracture group at 90 days, and limits the scope of our functional outcomes results at that time point. We found that participants with missing data had similar baseline characteristics to those with fully available data, potentially limiting the impact of these losses on the

\begin{tabular}{|c|c|c|c|c|}
\hline \multicolumn{5}{|c|}{ ADJUSTED for: age, sex, injury mechanism, presence of COPD or asthma and correlation of data } \\
\hline PHS 90 days & Differences & Lower & Upper & $p^{*}$ \\
\hline Sternal fracture $v$. Rib fracture & $-8,62$ & $-30,05$ & 12,82 & $<0.0001$ \\
\hline Sternal fracture $v$. No fracture & $-11,70$ & $-32,60$ & 9,19 & \\
\hline Rib fracture $v$. No fracture & $-3,09$ & $-9,80$ & 3,63 & \\
\hline
\end{tabular}



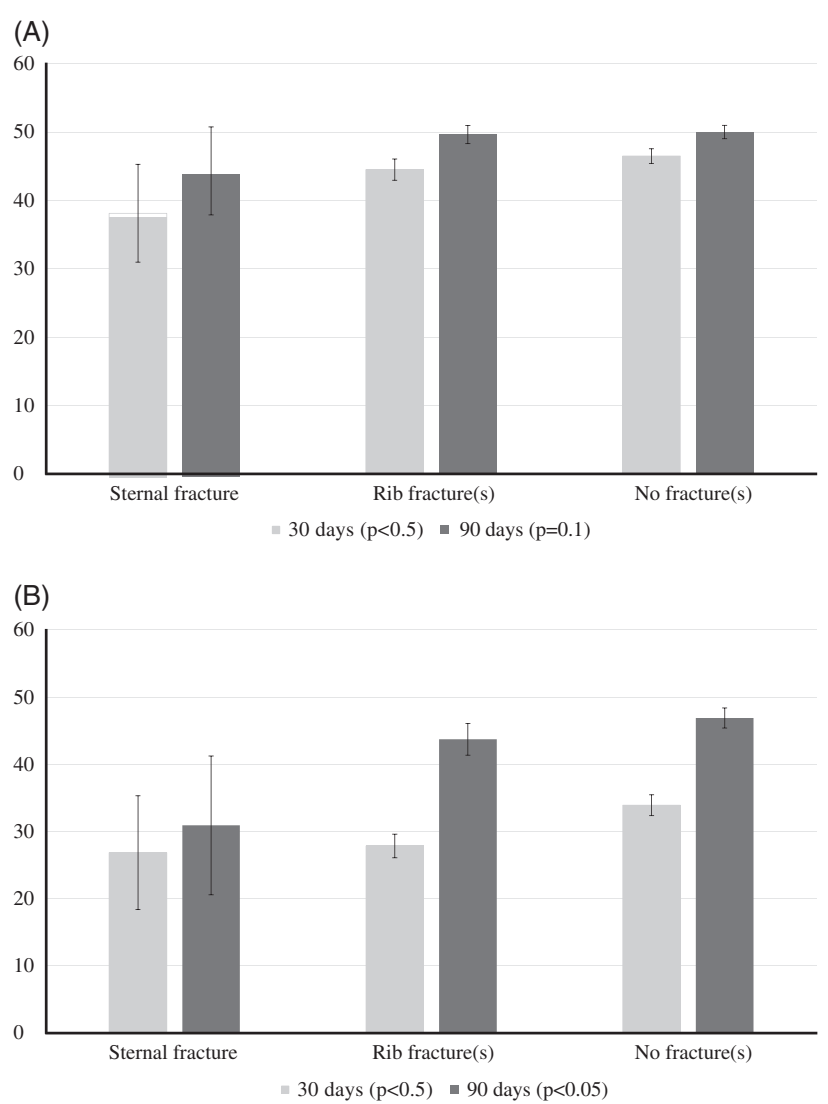

Figure 3. Physical functioning in daily activities and role limitations due to physical health problems at 30 and 90 days. A) Physical functioning in daily activities at 30 and 90 days. B) Role limitations due to physical health problems at 30 and 90 days.

results at 90 days. We feel that, as a result of our study protocol and logistics, it remains unlikely that patients lost to follow-up had major problems. Another limitation is that our participants' pre-injury functional status was not evaluated at their initial visit. This is known to be an important factor for the post-injury functional outcome and should be considered in the interpretation of our results. For this reason, our statistical analysis SF-12 scores were normalized for age and sex to minimize confounding from these factors. The fact that any visible pleural fluid on the chest $\mathrm{X}$-rays was deemed to be hemothorax is also a limitation that should be considered. There was no way, except drainage, to differentiate hemothorax from another effusion in our study. However, given the traumatic context, we felt it was reasonable to assume that any acute pleural fluid represented a delayed hemothorax. Finally, the fact that medical initial care and follow-up management were not standardized may also appear to some to be a limitation. However, our protocol that arose from this pragmatic design was intended to reflect as much as possible the actual care given in our EDs. The evaluation of the clinical importance of complications was left to the discretion of the treating physician and was not reported in our study for the same reason.

\section{CONCLUSIONS}

In this prospective study, we found that $12.5 \%$ of patients with sternal fracture developed a delayed hemothorax, but the clinical significance of this remains questionable. The proportion of patients with sternal fracture who had moderate to severe disability was significantly higher than that of patients with other minor thoracic trauma.

\section{CLINICAL IMPLICATIONS}

As a result of this new information that sternal fracture patients have more disability than patients with other minor thoracic traumas at 90 days, we feel clinicians should ensure that patients discharged from the ED with isolated sternal fracture have adequate analgesia and easy access to physician follow-up. Clinicians should also investigate for complications when assessing these patients during follow-up visits.

Acknowledgements: The results of this work were presented as a poster at the 31st Annual Meeting of the Association de Médecine d'Urgence du Québec and at the 9th Conference of the Département de médecine familiale et médecine d'urgence de l'Université Laval. We acknowledge Valérie Boucher from the CHU de Québec Research Center for editing and reviewing the manuscript and Brice Lionel Batomen Kuimi, MSc, who was in charge of the statistical analysis. We also thank Cong Tran, Marilyne Dufresne, Patricia Chabot, and Julie Lessard who have worked on data collection and day-to-day management, as well as the emergency physicians who participated in the recruitment of patients. SR was in charge of the literature review, data analysis and interpretation, and writing of the manuscript. Marcel Émond had full access to all of the data and takes responsibility for the integrity of the data and the accuracy of the data analysis. He was responsible for the design and conduct of the study. J-SA-C was involved in the literature search, review, and approved the manuscript. CG, LM, J-MC, NLS, EB, and LV were responsible for recruitment, and reviewed and approved the manuscript. This work was supported by a grant from Fonds de la Recherche en Santé du Québec (FRSQ 14056). This study has been approved by the Comité d'éthique de la recherche du CHU de Québec (institutional review board) (2012-1323).

Competing interests: None declared. 


\section{REFERENCES}

1. Knobloch K, Wagner S, Haasper C, et al. Sternal fractures occur most often in old cars to seat-belted drivers without any airbag often with concomitant spinal injuries: clinical findings and technical collision variables among 42,055 crash victims. Ann Thorac Surg 2006;82(2):444-50.

2. Recinos G, Inaba K, Dubose J, et al. Epidemiology of sternal fractures. Am Surg 2009;75(5):401-4.

3. Yeh DD, Hwabejire JO, DeMoya MA, et al. Sternal fracture-an analysis of the National Trauma Data Bank. 7 Surg Res 2014;186(1):39-43.

4. Bansal V, Conroy C, Chang D, et al. Rib and sternum fractures in the elderly and extreme elderly following motor vehicle crashes. Accid Anal Prev 2011;43(3):661-5.

5. Brookes JG, Dunn RJ, Rogers IR. Sternal fractures: a retrospective analysis of 272 cases. 7 Trauma 1993;35(1): 46-54.

6. Hossain M. 45-year-old male from an RTA with isolated sternal fracture: immediate discharge or hospital admission? Evid Based Med 2009;14(5):134-5.

7. Dua A, McMaster J, Desai PJ, et al. The association between blunt cardiac injury and isolated sternal fracture. Cardiol Res Pract 2014;2014:629-87.

8. Khoriati AA, Rajakulasingam R, Shah R. Sternal fractures and their management. 7 Emerg Trauma Shock 2013;6(2):113-6.

9. Kouritas VK, Zisis C, Vahlas K, et al. Isolated sternal fractures treated on an outpatient basis. Am 7 Emerg Med 2013;31(1):227-30.

10. Velissaris T, Tang AT, Patel A, et al. Traumatic sternal fracture: outcome following admission to a Thoracic Surgical Unit. Injury 2003;34(12):924-7.

11. Hossain M, Ramavath A, Kulangara J, et al. Current management of isolated sternal fractures in the UK: time for evidence based practice? A cross-sectional survey and review of literature. Injury 2010;41(5):495-8.
12. Misthos $\mathrm{P}$, Kakaris S, Sepsas E, et al. A prospective analysis of occult pneumothorax, delayed pneumothorax and delayed hemothorax after minor blunt thoracic trauma. Eur 7 Cardiothorac Surg 2004;25(5):859-64.

13. Plourde M, Émond M, Lavoie A, et al. Cohort study on the prevalence and risk factors for delayed pulmonary complications in adults following minor blunt thoracic trauma. CFEM 2014;16(2):136-43.

14. Émond M, Sirois MJ, Guimont C, et al. Functional impact of a minor thoracic injury: an investigation of age, delayed hemothorax, and rib fracture effects. Ann Surg 2015;262(5): 1115-22.

15. Kerr-Valentic MA, Arthur M, Mullins RJ, et al. Rib fracture pain and disability: can we do better? 7 Trauma 2003;54(6): 1058-63; discussion 63-4.

16. de Oliveira M, Hassan TB, Sebewufu R, et al. Long-term morbidity in patients suffering a sternal fracture following discharge from the A and E department. Injury 1998;29(8): 609-12.

17. Daoust R, Emond M, Bergeron E, et al. Risk factors of significant pain syndrome 90 days after minor thoracic injury: trajectory analysis. Acad Emerg Med 2013;20(11):1139-45.

18. Shields JF, Emond M, Guimont C, et al. Acute minor thoracic injuries: evaluation of practice and follow-up in the emergency department. Can Fam Physician 2010;56(3): e117-24.

19. Ware JE, Kosinski M. Interpreting SF-36 summary health measures: a response. Qual Life Res 2001;10(5):405-13; discussion 15-20.

20. Andrew NE, Gabbe BJ, Wolfe R, et al. Twelve-month outcomes of serious orthopaedic sport and active recreationrelated injuries admitted to Level 1 trauma centers in Melbourne, Australia. Clin 7 Sport Med 2008;18(5):387-93.

21. Jenkinson C, Chandola T, Coulter A, et al. An assessment of the construct validity of the SF-12 summary scores across ethnic groups. 7 Public Health Med 2001;23(3):187-94. 